\title{
HETEROGENEITY OF CASH MARKETS AT PHYSICAL DELIVERY POINTS AND THE HEDGING EFFECTIVENESS OF AGRICULTURAL COMMODITY FUTURES IN INDIA - LESSONS FOR CONTRACT OPTIMIZATION
}

\author{
SANJAY MANSABDAR*, HUSSAIN C. YAGANTI \\ Birla Institute of Technology and Science, Pilani, Hyderabad, India
}

* Corresponding Author: Sanjay Mansabdar, Department of Economics and Finance, Birla Institute of Technology and Science, Pilani, Hyderabad Campus, Jawahar Nagar, Hyderabad 500078, Indic

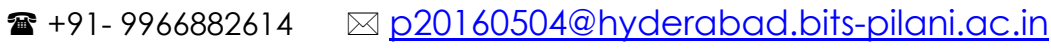

\begin{abstract}
Agricultural commodity futures in India are settled by physical delivery, and the seller can choose the location of delivery from a list described in the contract specifications. Cash markets at these locations represent the deliverable basket for the futures contract and are the underlying assets for the delivery options granted to the seller by virtue of contract design. These cash markets are generally heterogeneous. This paper studies the impact of heterogeneity of the underlying cash markets in different locations on the hedging effectiveness of the associated futures contract. The hedging effectiveness of cottonseed oilcake and soybean futures is regressed against several variables that represent heterogeneity of the underlying cash markets using ridge regression. We find that, in general, the greater the heterogeneity, the poorer the hedging effectiveness of the contract. This paper is unique in that it provides a framework for guidance for contract designers at exchanges and regulators who will find this research useful in optimizing delivery specifications for agricultural futures contracts. This is especially important given the declining volumes in Indian agricultural commodity futures.
\end{abstract}

Keywords: Delivery Options; Hedging Effectiveness; Agricultural commodity derivatives, Ridge Regression

\section{Introduction}

Hedging effectiveness is a key determinant of the success of futures contracts, as outlined by Tajishian (1995), Chance and Hemler (1993) and Yaganti and Kamiah (2012). Several studies have demonstrated that the hedging effectiveness of agricultural commodity futures contracts in India is in general poor. Yaganti and Kamiah (2012), for instance, suggest that only $40 \%$ of commodity derivatives are useful from a hedging effectiveness perspective. Aggarwal, Jain and Thomas (2014) find that the hedging effectiveness of agricultural commodities is low but variable. The problem is perhaps so acute that the Department of Economic Affairs, Government of India has set up a 
committee ${ }^{1}$ in the year 2017 to look into the issues causing the poor integration of cash $^{2}$ and derivative markets for commodities.

As Gulati, Chatterjee and Hussain (2017) have shown, the volumes of agricultural commodities futures in India has been on a decline since 2012, quite in contrast to volumes in other countries with agricultural commodity futures markets such as China and the US. The importance of hedging effectiveness for the success of futures contracts suggests that one could look for clues for this poor performance in diminished hedging effectiveness of such contracts. The data do appear to show that hedging effectiveness of agricultural futures has generally been on the decline over the period studied by Gulati et al., (2017). There has been a concomitant increase in the number of delivery locations for many contracts such as cottonseed oilcake (increased from 2 to eventually 6) and soybean (from 6 to eventually 9). Figures 1 and 2 show this trend for cottonseed oilcake (cocud) and soybean.

In Figure 1 and Figure 2, the moving average of hedging effectiveness over twelve expiries has been plotted along the left $y$-axis, with the number of deliverable locations for each expiry on the right $y$ axis with contract expiration on the x-axis (in YYYY-MM format). The moving averages convey the trend better as the hedging effectiveness data are noisy. The declining trend of hedging effectiveness and the concomitant increase in the number of locations over the same time period is evident, and the changes in direction also appear to be related. This suggests that perhaps the multiplicity of delivery locations along with some associated factors have a role to play in the declining hedging effectiveness of these contracts.

Figure 1: Evolution of hedging efficiency and number of delivery locations by contract cottonseed oilcake

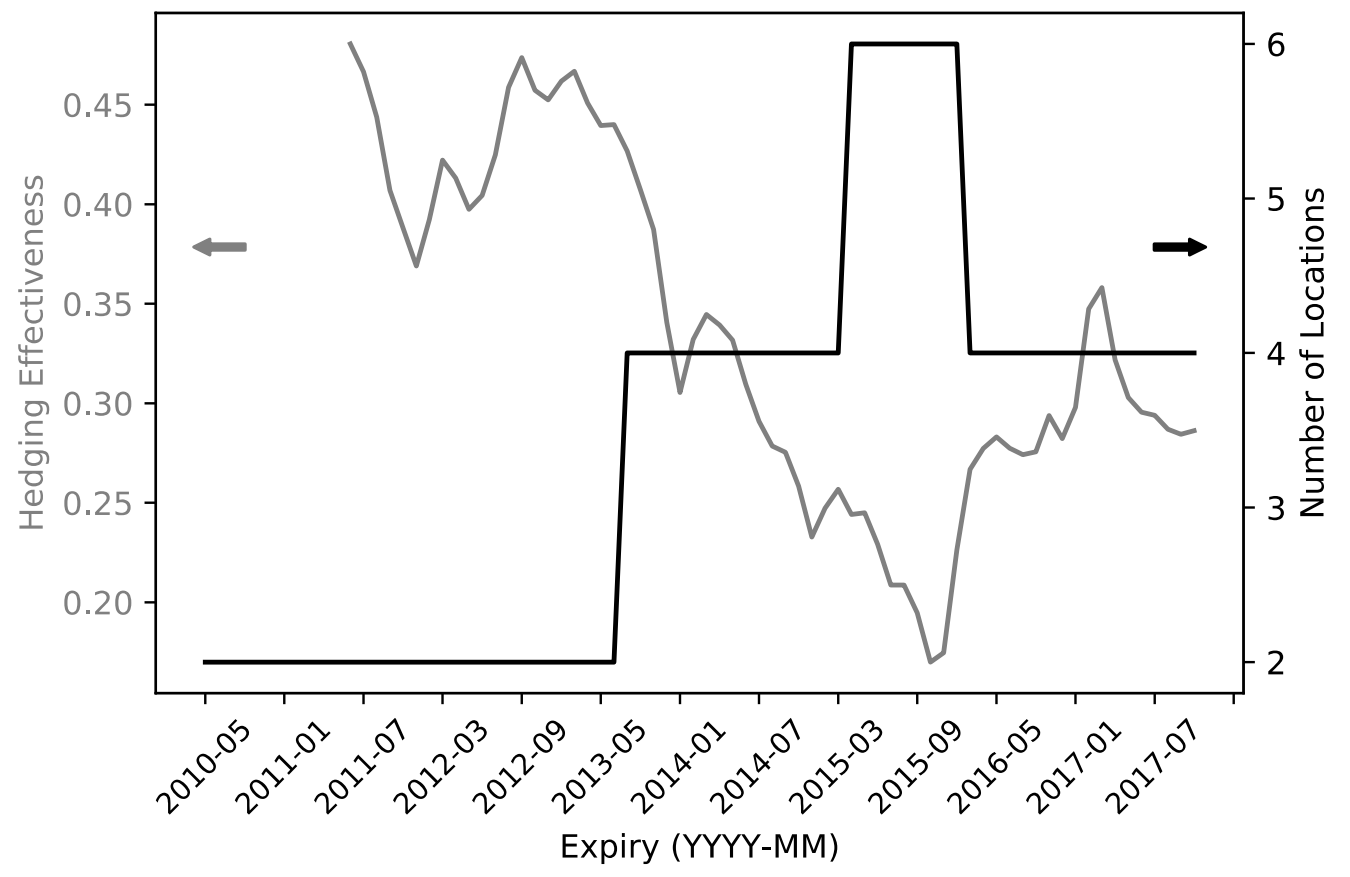

1 This committee was set up as a part of the Government of India's thrust on doubling farmer income by 2022.

2 In India cash markets are commonly referred to as spot markets. 
Figure 2: Evolution of hedging effectiveness and number of delivery locations by contractsoybean

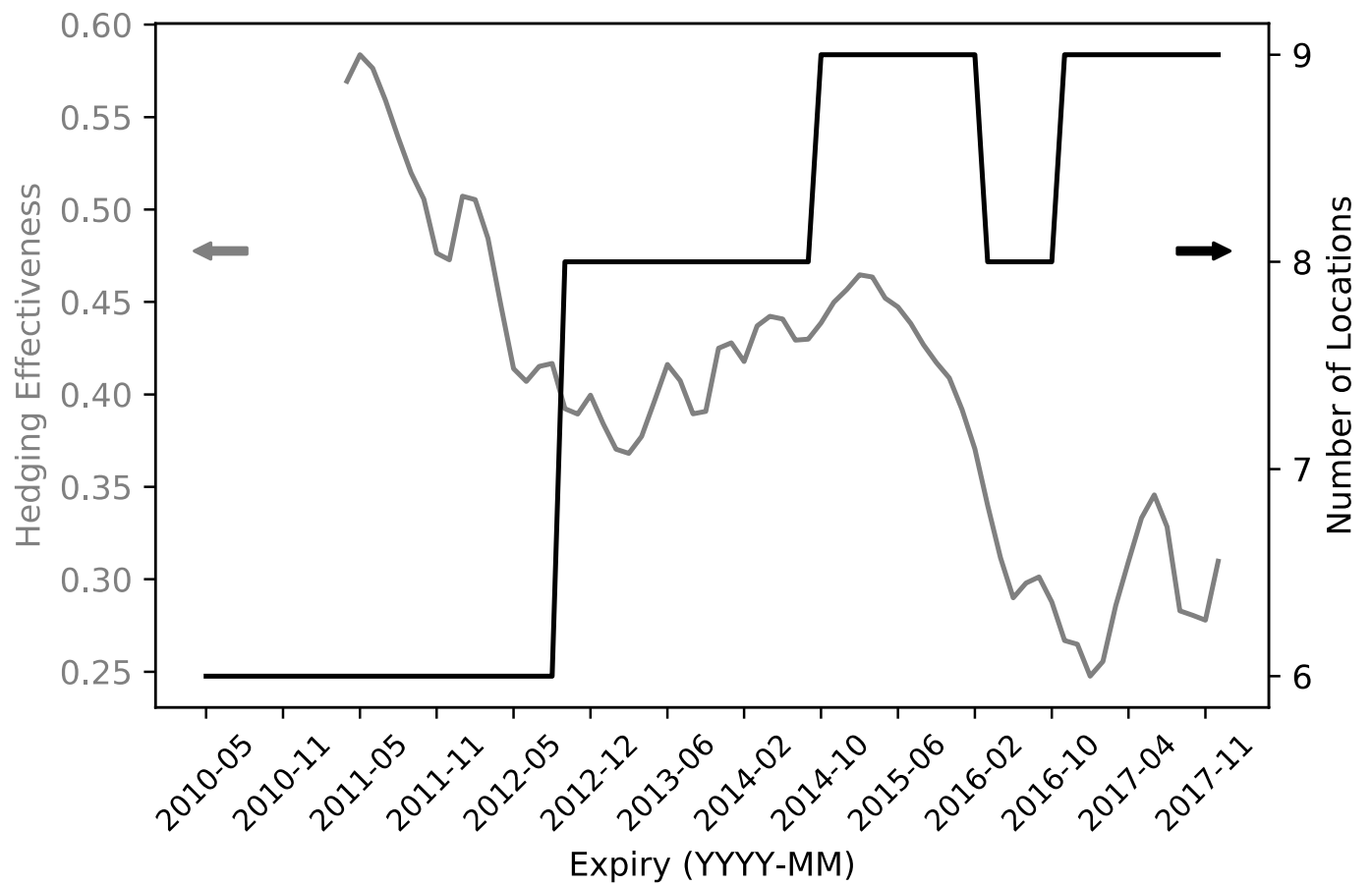

Agricultural commodity futures contracts in India are generally settled by delivery. The seller with an open position at expiration can choose to deliver at any of the delivery locations that are specified in the contract specifications. In other words, the very design of the contract furnishes the seller with an embedded ${ }^{3}$ option that allows him/her to decide where to make delivery. Cash markets for the underlying commodity exist at the different delivery locations permitted under the contract. The most important of these is generally chosen to be the reference asset or the par asset for the contract and hedging effectiveness of the futures contract is measured with respect to this cash market.

These deliverable cash markets, while being markets for the same underlying commodity, reflect local realities over and above the general conditions that determine the price of the commodity. For instance, it may be possible that for a commodity future with three deliverable locations $A, B$ and $C$ with $A$ being the par asset location, the underlying commodity traded in the cash market at $C$ has a higher moisture content as compared to that at location A due to agro-climatic factors, and hence may be of a slightly different grade. The commodity traded in the cash market in location B may be affected by local demand and supply factors that may lead to a different volatility and price change over a period of time as compared to the commodity traded in the cash market at A. Different regulations and taxes may apply to participants and markets at the different locations, leading to different conditions of demand and supply.

Such factors make the underlying cash markets heterogenous. It is generally assumed that the futures contract has a specific, narrowly defined commodity as the underlying reference asset in order to facilitate proper pricing and hedging. With many deliverable locations, the uncertainty with respect to what the underlying commodity is increases, and this brings about a change in standard no arbitrage pricing models for the future, which impacts its hedging effectiveness. The economic

\footnotetext{
${ }^{3}$ These are embedded in the sense that the delivery option is traded as a package with the future they are associated with.
} 
mechanism via which this happens are the embedded delivery options that the seller has, one of which is the location option described above. The inclusion of delivery options tends to reduce the value of futures contracts (Boyle, (1989)), which then reduces hedging effectiveness. Tajishian \& McConnell (1989) have shown that excessively high delivery option values are accompanied by impaired hedging effectiveness for a financial futures contract. This impaired hedging effectiveness led to the demise of the contract. It stands to reason from the above arguments that the greater the optionality, the greater the impact on hedging effectiveness. Many studies have highlighted the importance of the number of deliverable assets and their inter-se correlations on option values. Boyle (1989) has used order statistics to theoretically value the location option when there are a varying number of equi-correlated assets with identical starting prices and volatilities and finds that the greater the number of assets and the poorer the correlations, the higher the value of the options, and hence the greater the expected impact on hedging effectiveness. In a more general case, the relative values of the deliverable assets, their inter-se correlations and volatilities will tend to affect option values as shown by Boyle and Tse (1990), with its concomitant impact on hedging effectiveness.

It should be noted that the notion of heterogeneity as described above is strongly related to these ideas - the greater the number of deliverable assets, the greater the expected heterogeneity and the poorer the expected correlations between the deliverable assets, and the greater the value of embedded options. While the option valuation formulation suggests a mechanism of evaluating the extent of impact to hedging effectiveness, it does not provide any direct guidance as to what specific heterogeneity factors affect hedging effectiveness via the mechanism. These specific heterogeneity factors are those which contract designers and regulators have control over, and hence the knowledge of such dependence can be used to inform and optimize contract design. Optimizing the delivery specifications can help, therefore, in reversing the declining volume trends for agricultural commodity futures in India. Motivated by these practical considerations, we study the impact of various heterogeneity factors on hedging effectiveness. While contract design is not an exact science, such an understanding may perhaps provide guidance. We use some variables that are logical candidates to represent heterogeneity of the underlying cash markets. We assess the variation of the hedging effectiveness of the futures contract with that of these variables.

There is not much in the literature by way of prior studies in the area. It is generally well understood (see Boyle (1989) and Pirrong, Kormendi and Meguire (1994)) that underlying delivery assets for a futures contracts need to be highly correlated, which would imply some requirement of homogeneity. Tajishian (1995) discusses the cash markets characteristics required for optimal contract design, focusing on the need for high hedging effectiveness and the form of physical delivery design. (Gulati, Chatterjee, \& Hussain, 2017) suggest some characteristics of contracts that may be successful in the Indian context based on macro commodity characteristics. The lack of literature in this area is probably a consequence of the fact that extended time series of prices in multiple cash markets pertaining to an underlying commodity are not easily available. The National Commodities and Derivatives Exchange (NCDEX), which has the largest market share (over $80 \%$ ) of agricultural commodities derivatives volume in India, has a robust process for collecting and disseminating cash prices (see NCDEX (2018)) in several locations for the same commodity. This offers a unique opportunity to study, among other things, the impact of heterogeneity of the underlying cash markets on hedging effectiveness.

The remainder of this paper proceeds as follows - Section 2 discusses the approach considerations for and the choice of variables that represent heterogeneity of the underlying cash markets. Section 3 discusses the methodology, data and its availability, Section 4 the results and Section 5 concludes and highlights some policy implications. 


\section{Variables representing Heterogeneity}

\subsection{Hedging Effectiveness}

We define hedging effectiveness in accordance the work of Ederington (1979). Hedging effectiveness is defined as the $R^{2}$ of the regression of returns of futures $\left(R_{F}\right)$ and the returns of the cash market in the par asset location $\left(R_{S, A}\right)$. In other words, it represents the coefficient of determination of the regression model

$$
R_{F}=\alpha R_{S, A}+\beta
$$

The par asset defines the underlying reference asset for the commodity future and is, in general, the most important of the underlying deliverable assets. Delivery of any asset different from the par asset invites adjustments to the settlement price called premiums and discounts that are set with reference to this par asset. The benefits of addition of delivery locations should be weighed against the concomitant degradation of hedging effectiveness with respect to the par asset location. While futures contracts could be designed to optimize hedging effectiveness across some combination of delivery locations, presumably, the par asset location is chosen as such because it is the most important location when viewed in terms of number of participants, trading volumes, warehousing capacity and so on. Hence, at a minimum, hedging effectiveness at such a location should be high, to ensure success of a contract. We use hedging effectiveness as our dependent variable.

\subsection{Heterogeneity}

Studying the impact of heterogeneity of underlying cash markets requires defining alternative variables that represent heterogeneity of the underlying commodity market. In this regard it is important to clarify that it is the heterogeneity of the deliverable cash markets for underlying commodity that is being considered, i.e. assessing how different they may be within themselves. While there is no single parameter or variable that measures heterogeneity, it is instructive to look for available data that pertain to the differentiation between the various underlying cash markets as measures of heterogeneity. We consider the following candidates:

\subsubsection{Number of delivery locations}

The greater the number of delivery locations, the greater the possibility of heterogeneity of delivery location cash markets as discussed in Section 1.

\subsubsection{Number of states in which the delivery locations are geographically located}

In India agriculture is a state subject, with each state responsible for setting the regulations pertaining to the trading of agricultural commodities. These regulations could have different implications for the price behaviour of the local cash market. For instance, some governments have in the past restricted holding stocks beyond a maximum quantity. The consequent rush to liquidate inventories to ensure compliance has caused local cash markets to decouple from the general price trend of the commodity. The greater the number of states in which deliverable locations are situated, the greater the expected heterogeneity of the cash markets.

\subsubsection{The maximum distance between the delivery locations}

Commodities are expensive to transport and hence such costs act as friction in the transmission of a price change in one cash market to others. However, absent other transaction costs, once the price differential between two delivery locations is greater than transportation cost, it should be profitable to arbitrage by shipping the commodity from the lower priced location to the higher priced location. The greater the transportation cost, the greater the possible non-arbitrageable divergence in the prices at the cash locations. The greater the distance between locations, the greater the anticipated difference in behaviour of the cash markets. The maximum distance between two delivery locations is used as another variable representing heterogeneity of the underlying commodity. 


\subsubsection{Maximum volatility difference}

It appears reasonable to expect that the greater the difference in volatility, the greater the heterogeneity of the underlying commodity.

\subsubsection{Maximum price difference}

If cash prices in the underlying markets diverge significantly, the heterogeneity of the underlying commodity is likely to be high. Exchanges use premiums and discounts to account for price differences, so the prices used in computing these differences are actual cash prices adjusted by the premium/discount. Where a delivery location is accorded a discount, cash prices are adjusted upwards by the discount and where a delivery location is accorded a premium, the cash prices are adjusted downward by the amount of the discount. The maximum difference in the adjusted underlying cash market prices is used as a representation of heterogeneity. See Pirrong et al., (1994) for details on the use of premiums and discounts by exchanges and the adjustment of cash market prices. In general, a seller who delivers the cheapest to deliver instead of the par asset would be expected to realize a gain of

$$
S_{1, T}-\min \left(S_{1, T}, S_{2, T}+d_{2}, S_{3, T}+d_{3}, \ldots, S_{n, T}+d_{n}\right)
$$

where $S_{1, T}, S_{2, T}, \ldots, S_{n, T}$ are the cash market prices in the $n$ deliverable locations, at the time of expiration $T$ and $d_{2}, d_{3}, \ldots, d_{n}$ are the discounts applicable for all non-par delivery locations. The subscript 1 represents the cash market at the par asset location and hence has no corresponding discount term. This leads to the idea that it is price differences between adjusted prices that are pertinent for this analysis. We use prices adjusted by the discount/premium in order to compute this variable.

\subsubsection{Proportion of delivery that takes place at the par asset delivery location}

It is to be expected that if delivery decreases in the primary delivery location pertaining to the commodity, the heterogeneity of the commodity is likely to greater, as other delivery locations attain greater prominence at the expense of the primary delivery location. If this happens, clearly there are reasons for more than one underlying cash asset to become attractive to participants from a delivery perspective, indicating increased heterogeneity.

Our initial hypothesis is that for each commodity, the hedging effectiveness is dependent on each of these six heterogeneity variables with an inverse dependence on the first five and a positive dependence on the last.

\section{Methodology and Data Sources}

We recognize that contract design is based on judgment that considers several different variables. Specifically, the choice of delivery locations pertaining to a contract settled by delivery will depend on several variables, some of which have been identified above. There may be several others for which data is unavailable.

\subsection{Estimation Technique}

Our goal in this paper is to provide some guidance for contract designers to consider the heterogeneity of the underlying commodity cash market via the variables listed above. In assessing the impact of each of the above variables, it is important to recognize that these variables suffer from multicollinearity. To account for this, we use ridge regressions, a technique that is often used in such situations. The advantage over using this over other techniques such as principal component analysis in this situation is that the original observable variables are not transformed into others that are difficult to relate to. Given the goal of this paper, we believe that this is an important 
consideration. Ridge regression requires the selection of an optimum ridge ${ }^{4}$ parameter, for which several approaches exist. We use the methodology of Cule and De lorio (2012), which has the advantage that the ridge parameter is chosen automatically by controlling the variance of the model predictions. Our initial hypothesis suggests that the model for each commodity is:

$$
h=\sum_{i=0}^{n} \beta_{i} V_{i}+\alpha
$$

where $h$ is the hedging effectiveness of a futures contract, $V_{i}$ represent the various heterogeneity variables, $\beta_{i}$ represent the corresponding regression coefficients and $\alpha$ the intercept. We test whether the coefficients $\beta_{i}$ are statistically different from zero to assess whether the variable has an impact on hedging effectiveness.

\subsection{Data sources}

Publicly available data on the NCDEX website include the following:

- Cash Prices in the par asset location and some additional delivery locations

- Futures prices

- Premium and discount values for the locations where delivery is allowed

- Delivery values at each location per contract on expiration

We choose contracts that are highly liquid and figure in the top ten commodities traded on the NCDEX between the years 2010 and 2017, for which cash price data is available for the par asset location as well as at least one other delivery location. The years are chosen to reflect a combination of recency along with adequateness of data for the study. Additionally, we focus on commodities that show adequate variation in the variables listed in Section 1. Only soybean and cocud have adequate variability in the number of locations, with different contracts being deliverable at 6,8 and 9 locations and 2, 4 and 6 locations, respectively. For other commodities, the number of delivery locations change only by one, and hence it is difficult to draw any conclusions from the data. For cocud and soybean the other variables also vary in reasonable ranges and hence these two commodities are chosen for study. Since for the two chosen commodities the number of states does not change at all, we drop this variable on account of lack of data, even though we believe that such a variable should strongly reflect heterogeneity of the underlying commodity market.

The variables are calculated from the publicly available raw data as described above as follows:

1. Number of delivery locations $\left(V_{1}\right)$ : these are directly observed from contract specification for each expiry.

2. Maximum Distance between locations $\left(V_{2}\right)$ : The delivery locations were geocoded, and a distance formula applied to calculate the pairwise distance between delivery locations. The maximum of these was taken as the variable value.

3. Maximum volatility difference $\left(V_{3}\right)$ : cash prices for the delivery locations (where cash prices are available) were obtained for the two calendar months prior to the commencement of the expiry month. Given the bidirectional causality that is seen between futures and cash price changes, we consider, for a given futures contract, cash prices during the time that the futures contract is most liquid. This period comprises the two calendar months prior to the expiry month of the future, on account of the fact that as a contract enters the expiration month, the exchange imposes stringent position limits and margins, which lead to lower liquidity. Daily returns were calculated from these cash price series, and volatility of these

\footnotetext{
${ }^{4}$ The ridge parameter is variously referred to as the penalty or suppression parameter
} 
series were computed. Pairwise differences between these volatilities were calculated, and the maximum difference was chosen as the value of the variable for a particular contract.

4. Maximum price difference $\left(V_{4}\right)$ : As discussed in Section 2, prices are first adjusted for the exchange specified premium/discount. The pairwise adjusted price differences on each day in the two calendar months preceding the contract expiry month are calculated, and the maximum of these is chosen as the daily maximum difference. The maximum of all the calculated daily maximum differences is the value if this variable for a given contract.

5. Par asset delivery proportion $\left(V_{5}\right)$ : From contract level delivery data, the quantity of commodity delivered in the par asset location as a proportion of total delivered quantity across all delivery locations is taken as the value of this variable. Where the total delivery value is zero, the proportion is also taken to be zero.

For variables $V_{3}$ and $V_{4}$, data are incomplete in that time-series data for only a subset of all the delivery locations is available. We compute these variables on the basis of available data. Hedging effectiveness $(h)$ is calculated, as explained in Section 2. Par asset cash market returns and futures returns are calculated for the two months in which the futures are expected to be the most liquid as explained for the variable "Maximum Volatility Difference" above. Based on our hypothesis that increased heterogeneity is a cause of degraded hedging effectiveness, it is our expectation the regression coefficients for $V_{1}, V_{2}, V_{3}$ and $V_{4}$ are negative and the coefficient for $V_{5}$ is positive.

\section{Results and discussion}

The results for cocud and soybean are presented in Table 1 and Table 2 respectively.

Table 1: The impact of heterogeneity variables on the hedging effectiveness of cottonseed oilcake (cocud) futures. Correlation coefficient $=0.679$, ridge parameter $=0.1133$

\begin{tabular}{lcccc}
\hline Variable & $\boldsymbol{\beta}$ & scaled $\boldsymbol{\beta}$ & Standard Error & p value \\
\hline Number of locations $\left(V_{1}\right)$ & -0.0334 & -0.3901 & 0.1469 & $0.0079 * *$ \\
Max distance between locations $\left(V_{2}\right)$ & -0.0006 & 0.25118 & 0.1499 & $0.0939+$ \\
Max volatility difference $\left(V_{3}\right)$ & -0.0752 & -0.0323 & 0.1334 & 0.8088 \\
Max price difference $\left(V_{4}\right)$ & -1.5937 & -0.5447 & 0.1326 & $3.97 e-05^{* * *}$ \\
Par asset delivery proportion $\left(V_{5}\right)$ & 0.05368 & 0.1574 & 0.1253 & 0.2088 \\
\hline
\end{tabular}

Significance codes: ${ }^{* * * \prime}=0.0011^{\prime * * 1}=0.01$; $^{\prime * 1}=0.05$; $^{\prime}+{ }^{\prime}=0.1$

Scaled $\beta$ refers to the regression coefficient when the variable is scaled such that it lies between 0 and 1 . This procedure is necessary for ridge regression.

Table 2: The impact of heterogeneity variables on the hedging effectiveness of soybean futures. Correlation coefficient $=0.637$, ridge parameter $=0.1534$

\begin{tabular}{lcccc}
\hline Variable & $\boldsymbol{\beta}$ & scaled $\boldsymbol{\beta}$ & Standard Error & $\mathbf{p}$ value \\
\hline Number of locations $\left(V_{1}\right)$ & -0.0050 & -0.0567 & 0.1411 & 0.6878 \\
Max distance between locations $\left(V_{2}\right)$ & -0.0005 & -0.4849 & 0.1382 & $0.0005^{* * *}$ \\
Max volatility difference $\left(V_{3}\right)$ & -0.0987 & -0.0373 & 0.1262 & 0.7674 \\
Max price difference $\left(V_{4}\right)$ & -1.1303 & -0.5899 & 0.1213 & $1.14 \mathrm{e}-06^{* * *}$ \\
Par asset delivery proportion $\left(V_{5}\right)$ & 0.0934 & 0.2004 & 0.1209 & $0.0975+$ \\
\hline
\end{tabular}

Significance codes: ${ }^{\prime * * * \prime}=0.0011^{* * * 1}=0.01 ;^{i^{* 1}}=0.05 ;{ }^{\prime}+{ }^{\prime}=0.1$

Scaled $\beta$ refers to the regression coefficient when the variable is scaled such that it lies between 0 and 1 . This procedure is necessary for ridge regression. 


\subsubsection{Number of locations}

It is seen that the greater the number of locations, the poorer the hedging effectiveness as indicated by negative coefficients for both cocud and for soybean. The $p$ value is significant only for cocud at the 1 percent level and does not appear to be significant for soybean. The coefficients indicate a 3.3 percent degradation in hedging effectiveness on addition of a location for cocud.

\subsubsection{Maximum distance between locations}

For both cocud and soybean there appears to be an inverse dependence of hedging effectiveness on the maximum distance between locations. The negative coefficients are statistically different from zero for soybean at a 0.1 percent significance level and cocud at a 10 percent significance level as indicated by the $p$ value. The result appears to suggest than when the maximum distance between locations increases by 100 kilometres, the hedging effectiveness appears to fall by 5 percent for soybean and 6 percent for cocud.

\subsubsection{Maximum volatility difference}

For both cocud and soybean an increase in the maximum volatility difference has a negative impact on hedging effectiveness. However, the coefficients do not appear to be statistically different from zero for either commodity.

\subsubsection{Maximum price difference}

For both cocud and soybean, an inverse dependence of hedging effectiveness on max price difference is seen. The $\mathrm{p}$ values are significant at the 0.1 percent level implying coefficients that are statistically different from zero for both commodities. A 1 percent increase in the maximum price difference seems to degrade hedging effectiveness of cocud contracts by 1.6 percent while the corresponding figure for soybean is 1.1 percent.

\subsubsection{Par asset delivery proportion}

Cocud and soybean contracts both exhibit similar behaviour in that the hedging effectiveness appears to be positively related to the par asset delivery proportion. Coefficients are statistically different from zero only for soybean at the 10 percent level. For soybean, a 10 percent increase in the par asset delivery proportion appears to increase hedging effectiveness by about 0.9 percent.

The regression coefficients do have the signs hypothesized, though they are significant in varying degrees.

\section{Conclusions and policy implications}

The results appear to favour the hypothesis that the greater the heterogeneity of the underlying commodity cash markets at the multiple permitted delivery locations, the poorer the hedging effectiveness of the corresponding futures contract. Regressions yield coefficients that are statistically significant for at least one commodity for all variables except for the maximum volatility difference variable, and the direction of the dependence is consistent across both commodities, as are relative orders of magnitudes of the coefficients and the correlation coefficients.

These results have important implications for policymakers, regulators, and exchanges. For exchanges and regulators, providing contracts that deliver adequate hedging performance is a critical aspect in ensuring their acceptance by market participants and their consequent success. These results provide a framework for optimizing contract design and suggest that heterogeneity of the underlying deliverable commodities needs to be reduced or kept to a minimum to maximize hedging performance. The heterogeneity variables used in this study point to some concrete guidelines that can be followed in this endeavour. Specifically, 
- the number of delivery locations should be kept as low as practicable, as indicated directly, as well as the fact that increasing the number of delivery locations will increase the chances that one of these many cash markets may diverge significantly from the par asset,

- the delivery locations should be as close as practicable to each other,

- the premiums and discounts should be adjusted frequently to minimize large price differences between the delivery locations, and

- conditions for ensuring that a large proportion of delivery happens at the par asset delivery location (such as adequate warehouse capacity, appropriate premium/discounts, and ease of transportation) should be put in place.

In the context of making sense of the declining volumes of agricultural commodity futures in India, these results may present some guidance. The addition of many locations as shown in Figure 1 and Figure 2, perhaps in an attempt to expand the appeal of the contract to clientele in diverse geographies, may have simply resulted in excess heterogeneity in the underlying deliverable cash markets, thereby degrading hedging effectiveness. Such an approach at trying to make the contract work for many may have resulted in a situation where it has not worked for anyone consistently over the medium term.

Many of these results have intuitive appeal from the perspective of adjusting the impact of delivery optionality. By focusing on specific controllable variables, this study offers a framework for relating contract optimization to specific design parameters. Taken together with ongoing monitoring of delivery option values and hedging effectiveness, the framework offers a compelling vision for the design and ongoing optimization of agricultural commodity futures contracts. Such an approach may ultimately provide the optimal balance between hedging effectiveness and heterogeneity arising from delivery options necessary to prevent manipulation and squeezes, with a concomitant reversal in the trend of declining volumes in Indian agricultural commodity futures.

For policymakers such as the Department of Economic Affairs in the Ministry of Finance, Government of India, these results point to hitherto unrecognized areas that contribute to the problem their committee had attempted to resolve. The report of this committee (see Expert Committee on the Integration of Commodity Spot and Derivative Markets (2018)) does not appear to have considered the impact of contract design based on physical delivery on hedging effectiveness of futures, which creates a direct link between cash and derivative markets. These results suggest that some solutions for the problem of poor integration between cash and derivative markets that require only coordination with regulators and exchanges in optimizing contract design and are relatively low hanging fruit in this endeavour. Finally, coordination with state governments to establish orderly cash markets that are as homogenous as possible may also yield positive results in this direction, and the report has made a similar recommendation.

Finally, these results suggest further lines of research. Firstly, the maximum price difference appears to be a significant determinant of hedging effectiveness. Clearly, these are dependent on the premium and discount set by the exchange. These premiums and discounts are likely to affect the par asset delivery proportion, as participants will deliver at a location that is most economically favourable to them. Research into setting these premia and discounts optimally will help exchanges optimize contract design further. Secondly, this study could be extended by defining hedging effectiveness more broadly to be a combination of hedging effectiveness at all the delivery locations to meet the goal of maximizing contract appeal. Finally, approaches that focus on integrating the impact these variables via valuing embedded delivery options are likely to help complete the continuous monitoring, and optimization framework suggested earlier. 


\section{References}

Aggarwal, N., Jain, S. \& Thomas, S., 2014. Do futures markets help in Price Discovery and Risk Management for Commodities in India? IGIDR Working Paper WP-2014-020.

Boyle, P. P., 1989. The Quality Option and Timing Option in Futures Contracts. The Journal of Finance 44(1), 101-113.

Boyle, P. P. \& Tse, Y. K., 1990. An Algorithm for Computing Values of Options on the Maximum or Minimum of Several Assets. The Journal of Financial and Quantitative Analysis 23(1), 215-227.

Chance, D. M. \& Hemler, M. L., 1993. The Impact of Delivery Options on Futures Prices: A Survey. Journal of Futures Markets 13(2), 127-155.

Cule, E \& De Iorio, M., 2012. A Semi-automatic Method to guide the choice of Ridge Parameter in Ridge Regression, arXiv:1205.0686v1 [stat.AP]

Ederington, L. H., 1979. The Hedging Performance of the New Futures Markets. The Journal of Finance 34(1), 157-170.

Expert Committee on the Integration of Commodity Spot and Derivative Markets, 2018. Report of Expert Committee on the Integration of Commodity Spot and Derivative Markets, URL:

https://dea.gov.in/sites/default/files/ReportCommodityDerivativeMarkets042018_0.pdf

Gulati, A., Chatterjee, T. \& Hussain, S., 2017. Agricultural Commodity Futures: Searching for Potential Winners. ICRIER Working Paper 349.

NCDEX, 2018. Spot Price Polling, URL: https://ncdex.com/MarketData/PolledPrices.aspx

Pirrong, S. C., Kormendi, R. \& Meguire, P., 1994. Multiple Delivery Points, Pricing Dynamics, and Hedging Effectiveness in Futures Markets for Spatial Commodities. The Journal of Futures Markets 14(5), 545-573.

Tajishian, E. J. \& McConnell, J. J., 1989. Requiem for a Market: An Analysis of the Rise and Fall of a Financial Futures Contract. The Review of Financial Studies 2(1), 1-23.

Tajishian, E. J., 1995. Optimal Futures Contract Design. Quarterly Review of Economics and Finance 35(2), 153-162.

Yaganti, H. C. \& Kamiah, B., 2012. Hedging Efficiency of Commodity Futures Markets in India. The Journal of Financial Risk Management 9(2), 40-59. 